\title{
Lapurdum
}

LAPURDUM Euskal ikerketen aldizkaria | Revue d'études basques |

Revista de estudios vascos | Basque studies review

$8 \mid 2003$

Numéro VIII

\section{Amoros sekvetuki dena}

\section{Jean Haritschelhar}

URL : http://journals.openedition.org/lapurdum/1104

DOI : 10.4000/lapurdum. 1104

ISSN : 1965-0655

Éditeur

IKER

Édition imprimée

Date de publication : 1 novembre 2003

Pagination : 233-241

ISBN : 9782867813436

ISSN : $1273-3830$

\section{Référence électronique}

Jean Haritschelhar, « Amoros sekvetuki dena », Lapurdum [En ligne], 8 | 2003, mis en ligne le 01 mai 2009, consulté le 04 mai 2019. URL : http://journals.openedition.org/lapurdum/1104; DOI : 10.4000/ lapurdum. 1104 


\section{Amoros sekretuki dena}

La comparaison entre cette poésie et la précédente Ezkonduien koplak (Lapurdum VII, p. 237-246) est particulièrement intéressante. En effet, les thèmes sont voisins, un adultère en pensée pour le premier poème, l'incapacité de se déclarer pour le second, la barrière du mariage et la barrière du langage qui empêchent toute rencontre éventuelle.

Certes, on y peut déceler quelques différences. Le premier poème commence par un distique cher à Bernat d'Etxepare alors qu'il est absent dans le second. Ezkonduien koplak était formé de deux groupes de quatre quatrains séparés par un sizain central. De la même manière Amoros sekretuki dena propose :

* un groupe de trois quatrains qui annonce l'incapacité du timide à se déclarer,

* un quatrain central qui imagine l'heureuse rencontre des deux amants dont on apprend qu'ils sont tous deux déjà mariés, ce qui nous renvoie au poème des mariés,

* un deuxième groupe de trois quatrains qui envisage l'échec de l'union éventuelle.

Enfin la versification des deux poèmes utilise le vers de quinze syllabes $(8+7)$ couramment employé par Bernat d'Etxepare.

\section{1- Timidité}

Andre eder jentil batek bihotza daut ebatsi ;

Hartzaz orhit nadinian, deus ere ezin iretsi,

Nik hura nola, nahi nuke hark banentza honetsi

Ezin benturatuz nago, beldur dakion gaitzi.

Dès cette première strophe le problème de l'amoureux transi, éperdu, est posé. Le premier vers, bien articulé dans ses deux hémistiches, nous donne la description de la dame et les ravages qu'elle a causés dans le coeur de l'amoureux. Certes, la description de l'objet aimé est brève, mais il n'en est pas autrement pour Bernat Etxepare ; elle tourne autour autour de deux adjectifs, l'un eder qui évoque la beauté physique, l'autre jentil l'aspect moral, celui de la noblesse, c'est-à-dire parée de toutes les vertus qui correspondent aux âmes bien nées.

Le second hémistiche est caractérisé par le mot final ebatsi qui marque bien l'intensité de l'amour qui frappe celui qui n'est plus lui-même puisque le coeur, centre 
même des sentiments, lui a été ravi, et, de ce fait, ne s'appartient plus. Il est, en quelques sorte, pour reprendre l'expression espagnole de la même époque, "enajenado", transporté en un autre, aliéné au sens premier du terme c'est-à-dire devenu autre, caractéristique de l'amoureux fou.

L'homme est à tel point obnubilé qu'il en vient à perdre tout appétit deus ere ezin iretsi, autant dire, selon l'expression commune, qu'il vit d'amour et d'eau fraîche. Mais cet amour qu'il voue à la gente dame est-il partagé ? C'est bien la question essentielle et non résolue, qui relève du désir nahi nuke et qui se joue dans l'opposition ergatif/absolutif nik/hura et son correspondant hark/ni, le ni se trouvant inclus dans l'auxiliaire banenza du verbe onhetsi, placé lui aussi en fin de vers.

L'explication réside dans le dernier vers de la strophe où chacun des deux hémistiches met en valeur la timidité de l'amoureux : ezin benturatuz nago qui confine même à la peur d'être repoussé ou de déplaire à la belle beldur dakion gaitzi. Ainsi le décor est parfaitement dressé dans cette strophe d'introduction.

\section{Mirail bat nik ahal banu hala luien donoa, \\ Neure gogoa nerakutson sekretuki han barna, \\ Han berian nik nakusen harena ere nigana, \\ Hutsik ezin egin nezan behin ere hargana.}

Cette strophe du miroir est certainement celle qui traduit le mieux la pusillanimité de l'amoureux. N'étant pas capable de déclarer sa flamme, il souhaite avoir un intermédiaire qui serait un miroir. L'objet est, en effet, symbolique depuis la plus haute antiquité. Il reflète, renvoie à chacun sa propre image et chacun peut alors se rendre compte de ce qu'il est en réalité dans son aspect physique. Mais ici l'amoureux souhaite (ahal banu) posséder un tout autre miroir, un miroir magique qui aurait un don l'éventuel luien marque bien que nous plongeons dans l'irréel一, celui de refléter l'âme, de dévoiler à l'autre les pensées intimes (neure gogoa nerakutson), et cela dans le plus grand secret, dans un tête-à-tête souhaité.

Toutefois, le miroir doit avoir une double fonction. Il ne sert pas seulement d'intermédiaire entre l'amoureux et sa belle, il doit aussi apporter la réponse. Aux sentiments (neure gogoa) exprimés à travers le miroir, doit répondre sur le même miroir (han berian) la manière dont la gente dame a reçu la déclaration d'amour. Ce n'est pas un miroir à deux faces, mais un objet relevant du fantastique, de l'impensable, capable d'organiser le tête-à-tête le plus intime, véritable porte-parole d'un timide incapable de se déclarer. Le distique central de cette strophe dans une formulation en chiasme dont l'axe correspond aux verbes erakutsi/ikusi.

Neure gogoa nerakutson sekretuki han barna Han berian nik nakusen harena ere nigana 
suggère parfaitement cet aller-retour magique. Cela est fait pour éviter toute erreur éventuelle et que la conduite de l'amoureux soit toujours d'une perfection absolue.

Hutsik ezin egin nezan behin ere hargana.

Ene gaizki penatzeko hain ederrik sortu zen,

Gaoaz eta egunaz ere gaizki nizi penatzen;

Harekila bat banadi bihotza zait harritzen,

Neure penen erraitera are eniz ausartzen.

Cette strophe clôt cette présentation de la timidité maladive. Les deux premiers vers sont axés autour du même ensemble verbal gaizki penatu placé dans le premier hémistiche du premier vers et en final du deuxième hémistiche du second, donc se répondant parfaitement pour faire comprendre les affres de l'amour qui agitent le timide invétéré. Ce verbe composé marque bien les tourments endurés, le verbe luimême penatu employé la première fois avec valeur de proposition finale, la seconde fois au présent et l'adverbe qui le précède dont la valeur superlative est indéniable puisque dérivé de l'adjectif gaitz qui a aussi le sens de grand, énorme, gigantesque. D'où l'excellente traduction de René Lafon : «cruellement souffrir ».

La cruauté du destin se manifeste dans la naissance de cette beauté -le verbe est au passé- sortu zen source de souffrance future gaizki penatzeko et la permanence de cette souffrance gaoaz eta egunaz ere, puisque jour et nuit il est tenaillé, permanence marquée par le verbe au présent : gaizki penatzen.

De la perspective de la personne destinée à faire souffrir l'autre, de ce trajet du passé au présent, on aboutit au présent du suppositif bat banadi, une éventualité qui souligne plus que jamais la timidité du jeune homme. En effet, le voilà littéralement pétrifié. Son coeur est tellement saisi par l'effroi qu'il se transforme en pierre : bihotza zait harritzen et qu'il est incapable de prononcer le moindre mot. Comment, dès lors, exprimer les sentiments qui l'animent, cette peine profonde qui l'étreint. Le dernier mot du dernier vers, le verbe dans sa forme négative eniz ausartzen accuse l'incapacité dans laquelle se trouve l'amoureux transi.

\section{2- Rêve}

Ene gogoa baliaki, maite bide ninduke

Ni errege balin banintz, erregina litzate 
Hura hala nahi balitz, elgarreki ginate

Haren haurrak eta eniak aurride oso lirate.

La strophe centrale est placée sous le signe de l'hypothétique. Trois phrases la composent, les deux premières occupant chacune un vers et la dernière s'étendant sur deux vers. Chaque phrase est composée de la même manière, commençant par une proposition au suppositif et la principale soulignant l'éventualité par un verbe au conditionnel.

Le premier vers attire l'attention à la fois par les deux verbes tous deux placés à la fin de l'hémistiche, le premier baliaki et le second à la fin du vers maite ninduke, mais aussi par la particule bide dans l'ensemble verbal maite bide ninduke. Cette particule a une valeur de probabilité. Il ne s'agit pas de certitude mais le probable va au-delà du possible et le sens de la particule est généralement plus affirmatif que dubitatif. Ainsi le rêve prend corps, il a tendance à devenir réalité et donc, si le miroir remplissait son office une fin heureuse serait envisageable.

Dès lors, la joie éclate dans le deuxième vers. Pourquoi ne pas pousser le rêve à son comble en joignant le complexe de puissance, roi et reine, à celui de bonheur ? Heureux comme un roi ! On confine au délire.

Tout dépend de la belle, ainsi que l'indique le troisième vers, celui de l'union des deux amants, ce nahi, désir ou vouloir de la gente dame auquel il reste suspendu.

Les trois premiers vers ont exactement le même rythme de la subordonnée suppositive dans le premier hémistiche, de la principale au conditionnel dans le second. Ce rythme répétitif a quelque chose d'envoûtant, de féérique avec lequel contraste le dernier vers qui s'étale sur toute sa longueur.

\section{Haren haurrak eta eniak aurride oso lirate}

et crée la surprise totale. Ainsi la gente dame est déjà mariée, et le timide a dû, un jour, vaincre sa timidité puisque lui aussi est marié et père de famille. Voilà qui nous renvoie au poème des mariés.

\section{3- Echec}

Balinetan nik banerro hari neure bihotza,

Eta gero balin balit respuesta bortitza,

Dardoak baino lehen liro erdira ene bihotza ;

Duda gabe eror nainde han berian hil hotza. 
L'imagination reste toujours en action. Pour le velléitaire, incapable d'agir, tout se passe dans la tête et dans l'imaginaire. Il va donc se risquer, mais toujours en pensées. Curieusement les marques du suppositif sont doubles dans le premier vers, tout d'abord dans la particule balinetan de sens dubitatif et ensuite dans le préfixe $b a \mathrm{du}$ verbe banerro, ce qui suppose une éventualité moindre. Cependant est envisagée aussi, au deuxième vers, la réponse négative de la dame, autrement dit la pire solution, celle de l'échec total, cette respuesta bortitza. Ne serait-ce que par le vocabulaire, le mot respuesta, nous place dans l'ambiance plutôt hispanique de la femme fière et noble, soucieuse de son honneur.

Dès lors, le rêve de l'union de la précédente strophe s'évanouit et si les deux suppositions (déclaration, réponse) s'étalent sur deux vers les conséquences rempliront les deux autres vers du quatrain avec, en premier, l'image du glaive qui lui percerait le coeur où l'auxiliaire du verbe erdira, à l'éventuel dans ce cas (liro), montre bien que toute la scène relève de l'imaginaire. Encore plus dans le dernier vers qui envisage la mort brutale, toujours au conditionnel, eror nainde. Le rythme de ce vers de quinze syllabes qui divise chacun des deux hémistiches en deux $(4+4 / 4+3)$ montre les phases succesives de la mort. La certitude d'abord duda gabe, l'action ensuite eror nainde suivie de la localisation han berian pour terminer le vers par le mot composé hil hotza, figurant la mort hil mais aussi le corps refroidi hotza, la raideur cadavérique. Le déroulement de cette strophe et son final brutal par le mot hil hotza, nous place au coeur d'un drame, celui de l'amoureux repoussé.

\section{Artizarrak bertzetarik abantaila darama ; \\ Halaber da anderetan ni penetan nuiena. \\ Hanbat da eder eta jentil, hartzaz erho narama, \\ Zorihonian sortu date haren besoan datzana.}

A nouveau surgit l'image radieuse de la femme aimée en silence. La comparaison avec l'étoile du berger qui, dans le firmament, l'emporte sur toutes les autres par son brillant, sa luminosité abantaila darama, démontre bien ce que peut être la dame pour le coeur de l'amoureux toujours souffrant. Mais il y a plus, car la métaphore de l'étoile suppose aussi son éloignement, son caractère inaccessible. L'étoile, c'est-à-dire l'être aimé, est toujours objet de plaintes de la part de l'amoureux car froide, lointaine, inabordable. Elle symbolise l'amour non partagé.

Le troisième vers retrouve les deux qualificatifs de la première strophe eder eta jentil agrémentés cette fois-ci de l'adverbe hanbat qui intensifie la beauté et la noblesse de la dame, de telle manière que l'amour débouche dans l'aliénation, bien soulignée par narama auxiliaire de mouvement du verbe erho, lequel marque ce parcours vers la folie. L'amoureux ne s'appartient plus, il est conduit vers son destin de souffrances. 
Le dernier vers est comme un aparté, une constatation dans laquelle perce le sentiment de jalousie à l'évocation de l'union intime entre la dame et son mari. A l'amoureux qui reste dans la peine penatzen nuiena s'oppose la destinée heureuse du mari zorihonian sortu date qui repose entre les bras de sa bien-aimée. Cette situation nous ramène à celle du poème des mariés où l'amoureux voyait celle qu'il aimait dans les bras de son mari :

Beriakin datzanian, enetako aizia

(Quand elle est couchée avec son mari il n'y a pour moi que du vent)

et imaginait une issue qui pouvait être fatale :

Beriagana jelosturik deseperatzer nintzan

(Jaloux de son mari, je faillis céder au désespoir)

Ene gogoa nola baita zuzen jarri hargana,

Haren'ere Iangoikoak dakarrela nigana,

Ene pena sar dakion bihotzian barrena,

Gogo honez egin dazan desiratzen dudana.

Etonnante prière que celle de cette dernière strophe où l'amoureux souhaite l'intervention de Dieu pour ramener la dame à d'autres sentiments. L'élan qui conduit vers l'objet aimé mérite un mouvement inverse que seul Dieu peut obtenir. Le verbe dakarrela souligne bien ce mouvement souhaité par l'amoureux. N'est-ce pas la preuve d'un amour fou, d'une aliénation qui débouche dans le blasphème ? Il faut bien être "hors de soi", inconscient, déraisonnable, pour s'imaginer que Dieu puisse favoriser un adultère. Oublié le miroir qui sert d'intermédiaire ! Il lui faut un complice d'une autre envergure capable de pénétrer au plus profond du coeur, bihotzian barrena et qu'ainsi soit réalisé le voeu le plus secret de l'amoureux transi.

Le dernier vers suppose un bouleversement psychologique total de la belle et gente dame puisque c'est de son plein gré, gogo honez, qu'elle se soumettrait aux désirs de son amant. Prière blasphématoire qui relève d'un rêve insensé.

Bernat d'Etxepare, dans la deuxième partie des Linguae Vasconum Primitiae, celle consacrée aux poèmes d'amour, développe un certain nombre de variations sur les relations entre hommes et femmes. D'emblée, dans Amorosen gaztiguia, il montre sa dévotion envers la Vierge Marie, symbole de l'amour "a lo divino" qu'il place nettement au-dessus de l'amour profane qui fut, semble-t-il, celui des erreurs de jeunesse. Dans Emazteen fabore, il s'élève contre la misogynie ambiante, en rappelant la somme de bonheur que la femme procure à l'homme, y compris dans l'acte d'amour. Ezkonduien koplak aborde le thème de l'adultère "en pensée" et non "en action" se terminant par des considérations morales sur l'amour insensé et l'amour aveugle. 
Amoros sekretuki dena où tout reste secret, intérieur, voit un amoureux transi, déjà marié, aimant à la folie une belle dame qui n'est pas de son rang puisque jentil et, éperdu, sombre dans l'aliénation. Dans ce poème, encore, la morale l'emporte.

Jean HARITSCHELHAR

Président d'Euskaltzaindia

IKER - UMR 5478 - CNRS

N.B.- La traduction du poème, placée en annexe, est tirée de la traduction française de l'oeuvre de Bernat d'Etxepare par René Lafon et publiée dans : Bernat Dechepare, Olerkiak, Edili, San Sebastián, 1968, p. 166-167. 


\section{Celui qui est amoureux en secret}

Une belle et gente dame m'a volé mon coeur. / Quand je pense à elle, je ne puis rien avaler. / Je voudrais qu'elle m'aimât comme je l'aime ; / Incapable d'oser, je crains de l'offenser.

Puissé-je avoir un miroir qui fût doué d'une telle propriété / Que je puisse y faire voir en secret ma pensée à ma belle / Et y voir moi aussi la sienne à mon égard, / De façon à ne jamais commettre de faute envers elle!

C'est pour me faire cruellement souffrir qu'elle née si belle. / La nuit, le jour, elle me fait souffrir cruellement. / Si je la rencontre, j'ai le coeur saisi ; / Je n'ose même pas lui dire mes souffrances.

Si elle savait ma pensée, elle m'aimerait sans doute : / Si j'étais roi, elle serait reine ; / Si elle y consentait, nous serions l'un avec l'autre ; / Ses enfants et les miens seraient absolument frères.

Si je lui disais ce qu'éprouve mon coeur / Et qu'ensuite elle me répondît avec rudesse, / Mieux qu'un dard ses paroles perceraient mon coeur ; / Sans nul doute, je tomberais sur place, raide mort.

L'étoile du berger l'emporte sur les autres ; / Telle est parmi les femmes celle qui me fait souffrir. / Elle est si belle et si gentille que cela me rend fou. / Il est né dans la chance, celui qui couche dans ses bras.

De même que ma pensée s'est dirigée droit vers elle, / Que Dieu amène aussi la sienne vers moi ! / Que ma souffrance pénètre au plus profond de son coeur, / Pour qu'elle veuille bien faire ce que je désire! 